\section{Tyrosinkinasehemmer im Vergleich}

Ponatinib, ein Tyrosinkinaseinhibitor (TKI) der dritten Generation, hat nicht nur eine höhere Aktivität als andere TKI, sondern ist auch noch gegen eine wichtige Mutation unempfindlich. Das schlägt sich bei der Behandlung von Patienten mit Philadelphia-Chromosom-positiver akuter lymphatischer Leukämie $(\mathrm{Ph}+$ ALL) nieder, wie der Vergleich zweier Phase-II-Studien zeigt.

110 Patienten mit frisch diagnostizierter $\mathrm{Ph}+\mathrm{ALL}$ wurden in zwei prospektive Phase-II-Studien aufgenommen. 63 von ihnen erhielten eine hyperfraktionierte Therapie mit Cyclophosphamid, Vincristin, Doxorubicin und Dexamethason (HCVAD) plus Dasatinib als FrontlineTherapie, 47 Patienten bekamen HCVAD plus Ponatinib.

Für den Vergleich wurde eine Propensity-Score-Analyse nach 1:1-Matching angewendet und eine inverse Wahrscheinlichkeitsgewichtung (IPTW) vorgenommen.
Nach dem Matching ergab sich eine ereignisfreie 3-Jahres-Überlebensrate von $69 \%$ unter HCVAD/Ponatinib und von $46 \%$ unter HCVAD/Dasatinib ( $\mathrm{p}=0,04)$ sowie eine 3-Jahres-Gesamtüberlebensrate von $83 \%$ unter HCVAD/Ponatinib und von $56 \%$ unter HCVAD/Dasatinib. erhielten, lag an Tag 21 signifikant seltener eine minimale Resterkrankung (MRD) vor. Auch war in dieser Kohorte häufiger ein dauerhaftes zytogenetisches Ansprechen bei vollständigem Ansprechen zu finden, ein umfassendes molekulares Ansprechen bei vollständigem Ansprechen sowie nach drei Monaten und ein vollständiges molekulares Ansprechen nach drei Monaten. Christina Berndt

Sasaki K et al. Hyper-CVAD Plus Ponatinib Versus Hyper-CVAD Plus Dasatinib as Frontline Therapy for Patients with Philadelphia Chromosome-Positive Acute Lymphoblastic Leukemia: A Propensity Score Analysis. Cancer. 2016;122(23):3650-6.
Bei Patienten, die HCVAD/Ponatinib

\section{kurz notiert}

Forschungsstandort Deutschland

Krebs durch Epstein-Barr-Viren +++ Das Protein BNRF1 von Epstein-Barr-Viren fördert die Krebsentstehung. Teilen sich EBV-infizierte B-Lymphozyten, so verhindert es den ordnungsgemäßen Ablauf der Teilung, sodass sich häufig mehr als zwei Spindelpole (Zentrosomen) bilden. Viren, aus denen BNRF1 entfernt wurde, beeinträchtigen die Chromosomenverteilung dagegen nicht. Das fanden Wissenschaftler vom Deutschen Krebsforschungszentrum und vom Deutschen Zentrum für Infektionsforschung (DZIF) heraus [Shumilov A et al. Nat Commun. 2017;8:14257].

red.

Neues zur DNA-Reparatur +++

Die Serin-ADP-Ribosylierung ist ein Mechanismus zur Proteinmodifikation, der jahrelang übersehen wurde. Forscher vom Max-PlanckInstitut in Köln haben nun entdeckt, dass die Serin-ADP-Ribosylierung eine Schlüsselrolle bei der DNA-Reparatur spielt. Dabei werden Serinreste durch PARP-1 und PARP-2 ADP-ribosyliert. Diese Serinspezifität wird durch den HistonPARylation-Faktor 1 (HPF1) übertragen. Die Serin-ADP-Ribosylierung ist eine verbreitete Proteinmodifikation, die auf verschiedene Substrate abzielt [Bonfiglio JJ et al. Mol Cell. 2017;65(5) 932-40].

Dosierung signifikant niedriger als bei der Standarddosierung (199 vs. $150 \mathrm{mg} / \mathrm{m}^{2}$; $\mathrm{p}<0,001)$. Unter der pharmakokinetisch geleiteten Dosierung sank der Anteil an Patienten mit einer Paclitaxel-Exposition über der therapeutischen Schwelle von $38 \%$ in Zyklus 1 auf $2 \%$ in Zyklus 6.

Das Ansprechen war in beiden Armen vergleichbar ( 31 vs. $27 \%$; $p=0,405$ ), ebenso das mediane progressionsfreie Überleben (5,5 vs. 4,9 Monate) und das Gesamtüberleben (10,1 vs. 9,5 Monate).

Somit verbesserte dieses Vorgehen das Nutzen-Risiko-Profil von Paclitaxel bei Patienten mit fortgeschrittenem NSCLC. Eine pharmakokinetisch geleitete Dosierung könnten besonders wertvoll sein, wenn die Patienten gebrechlich sind, extremes Körpergewicht, Leberfunktionsstörungen und ein erhöhtes Risiko für eine Neuropathie aufweisen. Judith Neumaier

Joerger M et al. Open-label, randomized study of individualized, pharmacokinetically (PK)-guided dosing of paclitaxel combined with carboplatin or cisplatin in patients with advanced non-small-cell lung cancer (NSCLC). Ann Oncol. 2016;27(10):1895902.
Blutuntersuchungen geben Aufschluss über Nebenwirkungen von Paclitaxel. tintherapie. Die mediane Paclitaxel-Enddosis war bei pharmakokinetisch geleiteter 